\title{
Actividad integradora y formación del licenciado en Ciencias de la Educación, evaluación de su gestión
}

Ana-Bertha Luna

\section{RESUMEN}

El objetivo del artículo es presentar resultados de la evaluación del estado de la gestión de la actividad integradora, implementada como innovación curricular en la formación del licenciado en Educación, en una universidad pública mexicana, ante un nuevo modelo educativo institucional, el Modelo Humanístico Basado en Competencias (MHIC), fundamentado desde la pedagogía de la integración. Bajo una metodología mixta y desde la percepción de los estudiantes, se determina a siete años de su implementación que la gestión de la actividad integradora como innovación curricular del MHIC, alcanza cerca del $50 \%$ de efectividad, se muestran aciertos alcanzados, contradicciones y necesidades a superar.

Palabras clave: actividad integradora, pedagogía de la integración, gestión educativa, competencias universitarias, México. 


\section{Atividade integradora e formação do licenciado em Ciências da Educação, avaliação de sua gestão}

\section{RESUMO}

O objetivo do artigo é apresentar resultados da avaliação do estado da gestão da Atividade Integradora, implementada como inovação curricular na formação do licenciado em Educação, em uma universidade pública mexicana, ante o novo Modelo Humanístico Baseado em Competências (MHIC), fundamentado na pedagogia da integração. Sob uma metodologia mista e partindo da percepção dos estudantes, se determina a sete anos de sua implementação que a gestão da Atividade Integradora, como inovação curricular do MHIC, tem cerca de 50\% de efetividade; percebe-se acertos alcançados, contradições e necessidades a superar.

Palavras chave: atividade integradora, pedagogia da integração, gestão educativa, competências universitárias, México.

The integrating activity in the training of the graduate in Education Sciences: an evaluation of its management

\section{ABSTRACT}

The purpose of this article is to present the results of the assessment of the management of the Integrating Activity implemented as a curricular innovation in the training of the graduate in Education in a Mexican public university, within the context of the new Humanistic Competency-based Model (Modelo Humanístico Basado en Competencias, MHIC), founded on the pedagogy of integration. Based on a mixed methodology and from the students' perception, seven years after its implementation it is possible to determine that the management of the Integrating Activity, as a curricular innovation of the MHIC, has about $50 \%$ of effectiveness; it shows the achieved goals, the contradictions and the obstacles that need to be overcome.

Key words: integrative activity, integration pedagogy, educational management, university competencies, Mexico. 


\section{Introducción}

La actividad integradora se considera una innovación curricular didáctica, pedagógica, epistémica y metodológica, que permite al estudiante de educación superior articular la teoría con la práctica y mediar la movilización de sus saberes en el desarrollo integral de su formación profesional, y poder alcanzar el perfil de egreso deseado. Roegiers (2010: 31) define la integración como "una operación mediante la cual se hacen interdependientes diversos elementos que estaban disociados al principio, con el objeto de hacerlos funcionar de una manera articulada y en función de una meta establecida".

El concepto innovación en educación contempla diferentes definiciones, Cruz y Croda (2017) lo relacionan con el de reforma, en este sentido hablar de una innovación o reforma educativa tiene que ver con una macro transformación, es decir, una serie de innovaciones contenidas en toda una estructura de cambios significativos en la forma de organizar, pensar y definir un fenómeno, que tiene que ver por un lado con las innovaciones que están directamente relacionadas con los procesos de enseñanza y aprendizaje correspondientes a las necesidades actuales de estudiantes, y un segundo enfoque relacionado con el currículum en el sentido amplio de los saberes, posicionamientos y perspectivas.

Aunado a esto, la formación profesional mediante el enfoque por competencias en las universidades, ha traído como consecuencia la revisión de sus modelos de formación profesional para impulsar su desarrollo, el análisis de la finalidad de las reformas educativas, que al interior de las instituciones de educación superior (IES) surgen para su transformación y evolución, plasmada en su misión y visión.

En el caso de la Universidad Autónoma de Tlaxcala (UATx), universidad pública mexicana, en el Plan de Desarrollo institucional del 2006-2010 se impulsa una reforma universitaria para transitar de un sistema departamental a un sistema facultativo en una primera fase, y a continuación, para ofrecer una educación de calidad, se propone la acreditación de programas de licenciatura y la certificación del total de sus procesos administrativos, así como la renovación de su infraestructura. La concepción de modelo universitario como proyección de lo deseable, emerge del análisis histórico del pasado, de la valoración del presente y de una visión estructurada del futuro universitario, para plantear la reforma propuesta en 2011 como nueva alternativa de formación profesional en la UATx, en el contexto de la formación profesional integral por competencias (Ortiz, 2015).

Así surge el Modelo Humanista Integrador basado en Competencias (MHIC) propuesto por Ortiz, paradigma innovador en la formación profesional, el modelo tiene como finalidad propiciar en los estudiantes una formación humanista integradora, cuyo soporte práctico se fortalece con algunas propuestas de política educativa internacional, nacional y local, al que se le da un enfoque basado en competencias. Este modelo, fundamentado como humanista integral y con un enfoque socioconstructivista y de didáctica nueva, con un enfoque filosófico principalmente fundamentado en Octavi Fullat y el pedagógico en Frida Díaz-Barriga y Ángel Díaz- Barriga, en un inicio se denomina: "Modelo Humanista Integrador", y de manera consensuada con la comunidad universitaria se diseñan los elementos que conforman dicho modelo, alineados a la misión y la visión institucional (Ortiz, 2015).

Como parte innovadora del MHIC se diseña la propuesta de implementación del proyecto integrador denominado en la institución: "Actividad Integradora" (AI), como complemento al desarrollo de la estructura curricular, mediante campos formativos; Ortiz define a la actividad integradora en la propuesta institucional del MHIC como:

una situación de aprendizaje diseñada por los docentes de las diferentes unidades de aprendizaje de un mismo semestre, para ser realizada por los estudiantes, con la finalidad de articular: conocimientos, 
habilidades y actitudes planteados en la malla curricular.

La actividad integradora implica tanto la conformación de equipos de trabajo de estudiantes, como de grupos colegiados de docentes por semestre que tienen como propósito primordial la integración de los conocimientos de las diferentes unidades de aprendizaje en un trabajo interdisciplinario (Ortiz, 2015: 63).

La actividad integradora tiene como función principal impulsar el trabajo colaborativo entre estudiantes, para enfrentar situaciones de aprendizaje y desarrollar competencias genéricas, específicas y transversales, propuestas en el perfil de egreso de cada uno de los más de 40 programas educativos (PE) de licenciatura existentes en la UATx.

A seis años de su implementación, en la UATx no existe un estudio formal que reporte la evaluación del estado de la gestión de la actividad integradora al interior de las facultades y su impacto en los diferentes programas educativos de formación profesional.

Por ello surge la siguiente pregunta de investigación: ¿La gestión de la actividad integradora en la formación de estudiantes de educación superior, funciona con el diseño y la estructura adecuada planteada en el modelo, en cuanto a planeación, evaluación y retroalimentación de sus procesos, para que contribuya y mejore el proyecto curricular del PE del licenciado en Ciencias de la Educación; su gestión permite el fortalecimiento en la adquisición de competencias en los estudiantes, en el contexto de desarrollo del modelo MHIC?

\section{Desarrollo teórico-conceptual}

El ingreso a la universidad para el estudiante de nivel medio superior es un desafío, comentan Pérez et al. (2013), las estadísticas mencionan altos índices de deserción en los estudios universitarios, en la que reducidos porcentajes de estudiantes alcanzan su meta. Los cambios vertiginosos propios de la educación superior a los que se enfrentan los estudiantes en su ingreso a la universidad se convierten en un reto para el estudiante de nuevo ingreso, por lo que son necesarios aspectos motivacionales y formativos que contribuyan a su autonomía en el aprendizaje, que les permitan conocer, comprender y transferir nuevos conocimientos para alcanzar las competencias deseadas en su ingreso y tránsito en la universidad.

En opinión de Gaeta (2006) y Pérez et al. (2013), la falta de autorregulación y autonomía en el estudiante, para favorecer su aprendizaje en estudios de educación superior, sobre todo en los primeros semestres para evitar la deserción y el rezago escolar y llegar a una graduación exitosa, han provocado que en la gestión académica de los programas universitarios se implementen innovaciones y estrategias que favorezcan la formación universitaria eficaz, dadas las exigencias de este nuevo siglo de la información y la tecnología. Para ser un profesionista reflexivo, crítico y autónomo se necesita que el estudiante sea partícipe de su propio aprendizaje, estar motivado y con habilidades para seleccionar y usar estrategias, conocimientos y la propia regulación de los procesos cognitivos, que en el futuro le permitan actuar con liderazgo académico en el ejercicio de su profesión, para este fin algunas instituciones han propuesto la implementación del proyecto integrador en la malla curricular de su formación profesional.

El desarrollo de las competencias personales y profesionales en la universidad es considerado por Núñez et al. (2006) como uno de los principales indicadores de calidad, y en el marco del Espacio Europeo de Educación Superior (EEES), es una necesidad y prioridad para este nivel educativo, con una mayor interacción entre estudiantes y docentes, y sugieren promover que el alumno asuma la calidad en la educación como un proceso de mejora continua, para lo que son necesarios cambios en la enseñanza, en el papel del alumno y el rol del profesor, en el diseño e implementación del currículum y en las metodologías diseñadas para las estrategias de evaluación. 
Como una respuesta a estas problemáticas surge, entre otros aspectos, el enfoque por competencias, para un mejor desarrollo del estudiante mediante el modelo curricular educativo; Coll dice al respecto:

una competencia es la capacidad para responder a las exigencias individuales o sociales o para realizar una actividad o una tarea [...] cada competencia reposa sobre una combinación de habilidades prácticas y cognitivas interrelacionadas, conocimientos (incluyendo el conocimiento tácito), motivación, valores, actitudes, emociones y otros elementos sociales y comportamentales que pueden ser movilizados conjuntamente para actuar de manera eficaz (Coll, 2007: 35).

Esto posibilita la tarea de aprender promoviendo el saber, saber hacer, saber ser y saber estar, de forma que la educación universitaria desempeñe un papel fundamental en la preparación efectiva para aprender a lo largo de la vida. La clave está en que estas competencias posibiliten la tarea de aprender, promoviendo los distintos saberes de su formación profesional.

Por esta razón, las instituciones educativas se han dado a la tarea de implementar en el diseño curricular innovaciones como las actividades integradoras, que denominan proyecto Integrador (PI), y que permiten el desarrollo del enfoque por competencias en los estudiantes.

La necesidad de dar sentido a los aprendizajes y la necesidad de responder al aumento de la cantidad y accesibilidad de la información en el campo de la educación, plantea Roegiers (2010) en su Pedagogía de la integración, tiene como consecuencia que la función tradicionalmente reservada al maestro, que consiste en transmitir conocimientos a sus alumnos, pierda cada vez mayor sentido; además, el impacto de la globalización en los intercambios de información, ha influido desde un punto de vista económico, político y social, a enfrentar decenas de culturas diferentes; igualmente, las situaciones de aprendizaje en las universidades con la incorporación de las nuevas tecnologías, se hacen cada vez más complejas, por lo que la contribución del enfoque por competencias se da como respuesta a los desafíos planteados, y contribuye esencialmente a la realización de la persona, a su inserción social o socioprofesional en los medios en que ha sido llamada a evolucionar. Y con la intención de aplicar este enfoque en el terreno de la pedagogía y desde el plano de la gestión educativa, principalmente en instituciones de educación superior, se habla de "gestión integrada" de un sistema, que integra diferentes dimensiones de la gestión: varias categorías de actores, varios tipos de problemáticas (administrativas, económicas, pedagógicas, psicológicas).

Una de las funciones del PI para Cevallos et al. (2016) y Kalimullin et al. (2016), es mostrar que los estudiantes adquieren un aprendizaje significativo amplio y fundamentado si se interrelacionan las competencias de diversas asignaturas, de lo contrario, si éstas se trabajan sólo de manera independiente, además de representar esfuerzo y trabajo adicional al alumno, no generan una experiencia de aprendizaje completa. Los proyectos integradores reúnen los conocimientos de varias materias y logran que el estudiante obtenga una competencia completa resolviendo una problemática en específico.

El PI como estrategia didáctica funciona en la formación de diversas profesiones; respecto de la experiencia desarrollada en la formación del psicólogo, Hewitt (2007) da cuenta de la habilidad del estudiante para la búsqueda de información, su integración al servicio de la solución de un ejercicio de investigación y de la capacidad interpretativa de los saberes específicos de la propia disciplina, con una proyección hacia lo integrado del saber, lo que le permite una formación conceptual, actitudinal y procedimental hacia los procesos de investigación, en la resolución de problemas, bajo una mirada ética y de responsabilidad social universitaria. 
Además de lo anterior, Hewitt (2007) propone que el PI se convierta en una estrategia didáctica de evaluación por procesos y una estrategia pedagógica de impacto social, que puede utilizarse como apoyo a las diferentes asignaturas cursadas por los estudiantes del programa, con el fin de desarrollar competencias para observar, planear, diseñar y realizar acciones sistemáticas y pertinentes para el logro de cambios innovadores en el entorno, y en el ejercicio ético de su profesión.

En la experiencia citada por Pareja (s.f.) en la formación de ingenieros en Colombia, el Proyecto Integrador se constituye en un punto de convergencia de las funciones misionales de la universidad: investigación, docencia y extensión, y consecuentemente representa el punto de confluencia entre las diferentes demandas institucionales con miras a potenciar la integración administrativa, académica e investigativa. El objetivo principal del proyecto integrador en la formación de ingenieros, es fomentar en estudiantes y docentes un espíritu investigativo que parta de la experiencia proporcionada por la realización de un trabajo en equipo, en el que se pone en evidencia la integración de los saberes relacionados con cada disciplina de estudio en un semestre específico, y es importante destacar, en su desarrollo y planificación, la realización de proyectos para dar cuenta del logro de las competencias obtenidas por medio de dos informes: el primero con una estructura de proyecto y el segundo un informe científico en forma de artículo de los resultados obtenidos.

Para Parra y Pinzón (2013), la principal dificultad en la implementación de PI en la Facultad de Ingeniería de la Fundación Universitaria Panamericana de Compensar, ha sido generar espacios de trabajo de pares, que permitan unificar criterios ante los estudiantes y lograr el compromiso del 100\% de los docentes, como actores principales de su gestión, ya que el PI es utilizado como estrategia formativa de integración de personas, que surge como respuesta a la necesidad de fortalecer competencias específicas, lecto-escritoras, investigativas y de emprendimiento, mediante la creación progresiva de una cultura de gestión de proyectos. Y concluyen que del seguimiento a la implementación del PI con estudiantes y en el aula, se presenta la necesidad de generar tiempos adicionales de asesorías y talleres especializados en gestión de proyectos e investigación, así como de incentivar la generación de redes de negocio y apoyo a los estudiantes para fortalecer sus proyectos y crear códigos de ética en su formación, y finalmente llevar el modelo de PI a nivel institucional para que sea implementado en las demás facultades de la universidad.

Experiencias como las mencionadas por Hewitt (2007), Pareja (s.f.), Parra y Pinzón (2013) y Cevallos et al. (2016), señalan - en sus postulaciones teóricometodológicas y resultados de investigaciones - aspectos que indican que en la gestión educativa de este tipo de innovaciones curriculares, como la estrategia del PI, aún hay un amplio camino que recorrer para su consolidación dentro de la malla curricular de los programas educativos de formación universitaria, ya que requieren de un alto compromiso y apoyo de toda la comunidad académica (directivos, docentes, administrativos y de servicios) en su gestión institucional, a fin de fortalecer las competencias de formación profesional (genéricas, específicas y transversales) dentro de proyectos situados en el contexto del mercado de trabajo y del campo de la investigación, con un alto desarrollo e impacto social, la mejora de los procesos educativos y de la propia infraestructura de las instituciones.

En resumen, la propuesta de la pedagogía de la integración de Roegiers (2010) muestra diferentes perfiles, entre los cuales se pueden mencionar: una integración didáctica, una pedagógica y una cognitiva, que se manifiestan en la articulación de la formación teórica y práctica; actividades tendientes a estructurar los conocimientos antes del aprendizaje; organización de conocimientos por medio de un proyecto; integración de diferentes conocimientos, en lugar de la disociación de los mismos en las 
estructuras cognitivas del alumno; una acción concertada de varios formadores o de varios docentes que intervengan ante un mismo grupo de alumnos (integración, interformadores o interdocentes), y la articulación de varios enfoques (sociológico, psicológico, económico y filosófico).

Para captar una situación de interdisciplinariedad, así como la movilización de capacidades en transversalidad, es decir, en una integración de contextos, coinciden Roegiers (2010), Kallimullin et al. (2016) y Sanders (2012) en que el término integración se aplica en el sentido de una coordinación armoniosa de diferentes acciones que se llevan a cabo para el desarrollo de mejores aprendizajes como estrategia didáctica bajo una metodología compleja centrada en los estudiantes.

Bajo estos antecedentes, conceptualizaciones y resultados de investigaciones acerca del Proyecto Integrador y la problemática mencionada en el contexto de la UATx, se plantea dar respuesta a la pregunta de investigación con el siguiente objetivo:

Analizar, valorar y determinar la efectividad de la gestión de la actividad integradora (AI) en su diseño y estructura, planeación, estrategias y mecanismos e instrumentos de evaluación y retroalimentación, en su función de contribuir a la formación del licenciado en Educación, en el contexto de desarrollo del modelo MHIC, en la Facultad de Ciencias de la Educación de la Universidad Autónoma de Tlaxcala.

\section{Estrategias metodológicas}

Se considera de primordial importancia la investigación de tipo evaluativa de la gestión de esta importante innovación curricular, la actividad integradora en su propio contexto de desarrollo, ya que involucra la formación de estudiantes de manera colaborativa y la formación y actuación de grupos colegiados de docentes que dirigen, supervisan y evalúan la actividad integradora, se pretende dar cuenta del funcionamiento de la gestión con fines de retroalimentación a través de los procesos formativos que se llevan a cabo en los estudiantes. La investigación se realizó bajo un enfoque mixto, se presentan parte de los resultados obtenidos bajo la técnica de encuesta con preguntas cerradas y abiertas, inicia como exploratoria, de tipo descriptiva y valorativa del desarrollo de la actividad integradora, desde la percepción de los estudiantes de la licenciatura en Ciencias de la Educación.

\section{Diseño del instrumento, validación y aplicación}

Diseño. Para la investigación bajo la técnica de encuesta se diseñó un instrumento tipo cuestionario en tres partes: la primera parte incluye variables categóricas independientes que determinan el perfil sociodemográfico de los estudiantes, la segunda parte integra un inventario de las herramientas tecnológicas y de servicios educativos de apoyo personales e institucionales, propios para la realización de su actividad integradora, con una escala de Likert de cuatro adjetivaciones, y por último, el tercer apartado del cuestionario corresponde a la parte valorativa de las percepciones de las variables dependientes del estudio con una escala de Likert de cinco adjetivaciones que van desde siempre hasta nunca para 37 ítems, que permiten analizar los logros, las contribuciones, motivaciones y el apoyo docente en el desarrollo de la AI, así como necesidades y situaciones de controversia y paradoja que se presentan en las actividades que llevan a cabo. El constructo se diseñó en seis categorías de análisis, y se presenta el resultado de tres de ellas: la formulación, el diseño y estructura de la AI incluida en el proceso de planeación; los factores didácticos y pedagógicos de impacto en la AI, y las estrategias y mecanismos de evaluación utilizados en su desarrollo.

Validación. Se valida el constructo en un grupo de cuatro docentes que han impartido la asignatura de la AI en más de dos ocasiones y de cuatro docentes especialistas en el tema que participaron en la elaboración del PE bajo el modelo MHIC; de manera 
cualitativa se validó relevancia, pertinencia, claridad y aspectos formales en cada uno de los 37 ítems de las seis categorías propuestas, y de manera cuantitativa, por medio de un análisis no paramétrico de Kolmogorov-Smirnov, con una significancia de 0.198 y Shapiro-Wilk con una significancia de 0.005 para validar la normalidad de los datos, así como mediante el estadístico alfa de Cronbach, que mide la fiabilidad del ítem, con un valor promedio obtenido de 0.898 para 37 elementos.

Población, muestra y sujetos. Para esta investigación se considera como población a los estudiantes de la licenciatura de Ciencias de la Educación, de la misma Facultad de la UATx, del $5^{\circ}, 6^{\circ}, 7^{\circ}$ y $8^{\circ}$ semestres inscritos en el periodo vigente primavera 2018, la muestra probabilística al $95 \%$ de confianza y $5 \%$ de margen de error, indicó 94 sujetos como mínimo para aplicar el instrumento; se tomaron en cuenta los siguientes requisitos de inclusión: pertenecer a alguno de los semestres mencionados y haber realizado la actividad integradora al menos dos veces.

Aplicación. Se aplicó el cuestionario por grupos, a los estudiantes que asistieron a los exámenes ordinarios y actividades de fin de semestre (primavera 2018), de manera intencionada, según los requisitos de inclusión y de libre participación, involucrando a los 94 estudiantes de ambos turnos: matutino y vespertino.

El análisis de los datos. Se realizó mediante los softwares SPSS V. 21 y Excel, un análisis de frecuencias y porcentajes para las variables independientes: sexo, edad y semestre; se presentan en la Parte I de resultados. Para las variables dependientes se llevó a cabo, por medio de un análisis de frecuencia relativa de la respuesta positiva, el cálculo del porcentaje de efectividad de cada categoría del constructo valorado y un análisis de inferencia estadística de comparación de medias para una muestra, mediante el estadístico T Student, para la comprobación de hipótesis de los ítems incluidos; los resultados se presentan en el apartado II, se incluyen tablas y gráficas.

\section{Actividad integradora y formación del licenciado en Ciencias de la Educación, su diseño, gestión y evaluación (resultados, su análisis y discusión)}

En este apartado se describen los resultados y su análisis en tres partes: la primera describe a los sujetos participantes por sus datos sociodemográficos, académicos y los recursos con los que cuenta el estudiante (variables independientes y categóricas), en la segunda se describe la valoración estadística del análisis de los datos obtenidos en la valoración del trabajo de campo (variables dependientes), y en la tercera la contrastación con los datos cualitativos de las preguntas abiertas.

\section{Parte I. El futuro docente y recursos disponibles en su formación académica}

El 30\% de los estudiantes participantes son de sexo masculino y el $70 \%$ de sexo femenino; por edad: los de 20 años o menos están agrupados en el 27\%, el $58 \%$ tiene de 21 a 23 años y el $15.5 \%$ de 24 a 26 años; todos cursaban quinto, sexto, séptimo y octavo semestres de la licenciatura en Ciencias de la Educación. De éstos, el 51\% cuenta con algún tipo de beca para la realización de sus estudios. El 92\% son estudiantes solteros. Con respecto a sus calificaciones, el $6 \%$ tiene un promedio de 10 ; el $50 \%$ de los estudiantes ha alcanzado un promedio de 9 a 9.9; el $35 \%$ mantiene un promedio de 8 a 8.9, el 8\% mantiene un promedio de 7 a 7.9. Estos datos sociodemográficos permiten visualizar una fortaleza, en su mayoría son estudiantes dedicados a sus estudios, en donde el 50\% cuenta con algún tipo de apoyo institucional, un mínimo porcentaje es casado, más del $80 \%$ mantiene un promedio superior a 8 . Lo que nos indica que son estudiantes con interés y enfocados en el estudio para llegar a ser estudiantes competitivos, en donde la AI juega un papel muy importante para la consolidación en su formación en competencias, citadas en el perfil de egreso.

Recursos disponibles. En cuanto al inventario realizado de recursos disponibles para la realización de 
sus tareas, el 70\% ha desarrollado la AI más de cuatro veces, el $80 \%$ tiene computadora e internet en casa, el 70\% cuenta con una laptop de uso personal, el 65\% cuenta con internet en su celular, el 60\% considera útil la biblioteca de facultad y el centro de cómputo en sus actividades académicas y solamente el 53\% considera adecuada la infraestructura de la facultad para la realización de sus tareas. Estos datos reflejan que la infraestructura que se ofrece en la facultad aún no logra satisfacer las necesidades de todos los estudiantes para los objetivos programados y es necesario entender y atender principalmente la situación de aquellos que no cuentan con los complementos propios para el desarrollo de la AI, esta situación establece una marcada desigualdad y una brecha entre estudiantes para su formación integral.

\section{Parte II. La efectividad de la actividad integradora en la formación del licenciado en Educación}

En este apartado y para este artículo, se describen parte de los resultados obtenidos en la valoración de las variables dependientes en el estudio, comprendidas en la gestión de la AI al interior de la institución: II.1. El diseño de los proyectos, su estructura y planeación, II.2. Los procesos didácticos y II.3. Los procesos, mecanismos e instrumentos de evaluación, implementados en el desarrollo de la actividad integradora.

\section{II.1. El diseño, la estructura y planeación de la actividad integradora}

El diseño y la planeación en el desarrollo de la AI adquiere relevante importancia en la formación de los profesionistas bajo el modelo de competencias, se valoraron aspectos como el diseño de objetivos; la estructura del proyecto para alcanzar la profundidad adecuada en la planeación de los contenidos, así como la congruencia presentada entre objetivos y contenidos; el trabajo colaborativo y la interacción realizada entre docentes y entre docentes y estudiantes, así como el logro de los conocimientos adquiridos.

Los resultados obtenidos por medio del cálculo del algoritmo que determina la frecuencia relativa de las respuestas positivas desde la percepción de los estudiantes, alcanzan en promedio un $49 \%$ de efectividad en el desarrollo de la AI, en un sistema de calidad, el logro del 50\% más uno determina que la implementación está en desarrollo, muestra una afinidad y desarrollo a lo planeado. El promedio alcanzado muestra una valoración media, sin embargo, es importante hacer notar los logros y esfuerzos realizados por los docentes y estudiantes para llevar a cabo esta actividad, el mayor porcentaje obtenido fue de $62 \%$ de respuestas a favor, logrado para la profundidad de contenidos manejados en la AI, mientras que la valoración más baja, con un 38\% de efectividad, fue para el trabajo colaborativo desarrollado entre estudiantes y profesores. Los resultados obtenidos muestran la necesidad de reflexionar sobre el rol del docente, su apropiación de los fundamentos de la pedagogía de la integración, de la planificación, del trabajo en colegiado y de la interacción lograda con los estudiantes, para dar instrucciones, seguimiento, supervisión y asesoría en el ejercicio de la AI. Es necesario que se muestre interés y se motive al estudiante para que perciba un trabajo colegiado y procesos de comunicación efectiva entre docentes y su interacción con los estudiantes, y solicitar el apoyo docente en caso de ser necesario.

Se valora también como un factor crítico, con un $43 \%$ de efectividad, la adquisición de las competencias profesionales a través de la realización de estos proyectos, que les permitan a los estudiantes adquirir nuevos conocimientos por medio de la AI. En aspectos como la valoración de la congruencia entre objetivos y contenidos diseñados, se obtiene una valoración de $43 \%$ de respuestas a favor, punto importante de reflexionar en el proceso de planeación, pues resulta paradójico para los objetivos que persigue la actividad integradora de alcanzar los propósitos y lograr la 
autonomía del estudiante, en donde la planeación es punto prioritario por atender; el promedio de efectividad alcanzado para esta dimensión es de $53 \%$, por arriba del mínimo aceptable 50\%.

La aplicación de la prueba T Student para una muestra (inferencia estadística) aplicado a las seis variables dependientes evaluadas en esta categoría, permite rechazar la hipótesis nula (Los factores de la planeación académica no cumplen con lo planeado en la AI en lo mínimo aceptable), resultados que coinciden con la medición de la efectividad de la planeación en el desarrollo de la AI. Los estudiantes valoran el desarrollo de estas seis variables en el nivel mínimo aceptable, en las que la congruencia entre los objetivos planeados y los contenidos de la AI y la colaboración desarrollada entre docentes y estudiantes se encuentran por debajo del promedio de la media entre variables igual a 3.07 (ver tabla 1 y gráfica 1).

Un correcto proceso de planeación educativa y colegiada de los docentes en el desempeño y ejercicio de la AI, logrará que los estudiantes cumplan sus objetivos y metas establecidas en el desarrollo de sus competencias, lo que será un reflejo de la calidad de educación recibida. Así, los cambios que están abordando las universidades llevan ese sentido concreto en su visión y su misión, con el firme propósito de que las universidades sean verdaderas instituciones promotoras del cambio para este siglo. Los nuevos profesionistas, como consecuencia, serán agentes de cambio, e innovadores en el contexto de la educación superior.

Como consecuencia de los resultados obtenidos en esta investigación que supera el $50 \%$ de efectividad, es importante resaltar los esfuerzos de los docentes para llevar a cabo el desarrollo de la AI, se coincide con las aportaciones de investigaciones realizadas en otras universidades, como la de Núñez et al. (2006) y Cevallos et al. (2016), quienes mencionan que cabe resaltar que los esfuerzos realizados por las comunidades académicas son de relevante importancia, ya que a pesar de las diferencias profesionales que existen entre los docentes que las integran, y las deficiencias que aún faltan de atender en la institución para lograr que en el corto plazo, por medio de la actividad integradora, se logran optimizar los principios

Tabla 1. Factores de incidencia en el diseño y la planeación de la AI

\begin{tabular}{|c|c|c|c|c|c|c|}
\hline $\begin{array}{l}\text { Número } \\
\text { de Ítem }\end{array}$ & Variable & T Student & Significancia & Media & $\begin{array}{c}\text { Interpretación al } \\
\text { valor de comparación } \\
\text { rechaza la Ho }\end{array}$ & $\begin{array}{c}\text { Efectividad } \\
\text { Alcanzada en la } \\
\text { implementación }\end{array}$ \\
\hline 3 & Planeación y diseño de objetivos & $5.95^{*}$ & 0.000 & 3.07 & *Mínimo aceptable & $53 \%$ \\
\hline 10 & Profundidad de contenidos & $5.394^{*}$ & 0.000 & 3.00 & *Mínimo aceptable & $62 \%$ \\
\hline 12 & Congruencia entre contenidos y objetivos & $6.563^{*}$ & 0.000 & 3.08 & *Mínimo aceptable & $48 \%$ \\
\hline 16 & Colaboración docente- estudiante & $4.355^{*}$ & 0.000 & 2.95 & *Mínimo aceptable & $38 \%$ \\
\hline 18 & Logro de objetivos & $7.418^{*}$ & 0.000 & 3.18 & *Mínimo aceptable & $49 \%$ \\
\hline \multirow[t]{2}{*}{21} & Nuevos conocimientos & $6.222^{*}$ & 0.000 & 3.18 & *Mínimo aceptable & $43 \%$ \\
\hline & Promedio & & & 3.07 & & $53 \%$ \\
\hline
\end{tabular}

Fuente: propia de la investigación

Interpretación: Excelente ${ }^{* * * * *} u=4.5$ a 5; Muy bueno ${ }^{* * * *} u=4-4.4 ;$ Bueno $^{* * *} u=3.5$ a 3.9; Regular $^{* *} u=3.0-3.4 ;$;ínimo Aceptable $u=2.5-2.9$. 
de la planeación didáctica en la práctica de la autorregulación del aprendizaje en los estudiantes, y que no debe ser entendida solamente como una aptitud mental, sino como un proceso de autodirección, a través del cual los alumnos transforman sus aptitudes mentales en competencias académicas en lo individual y en colaborativo, y que además presentan características como ser proactivos en cuanto a sus esfuerzos por aprender, ya que son conscientes de sus habilidades y limitaciones y, además, muestran comportamientos para el estudio guiados por objetivos y estrategias que los ayudan a alcanzarlos, con la supervisión de sus profesores.

\section{Gráfica 1. Factores de incidencia en el diseño y la planeación de la Al}

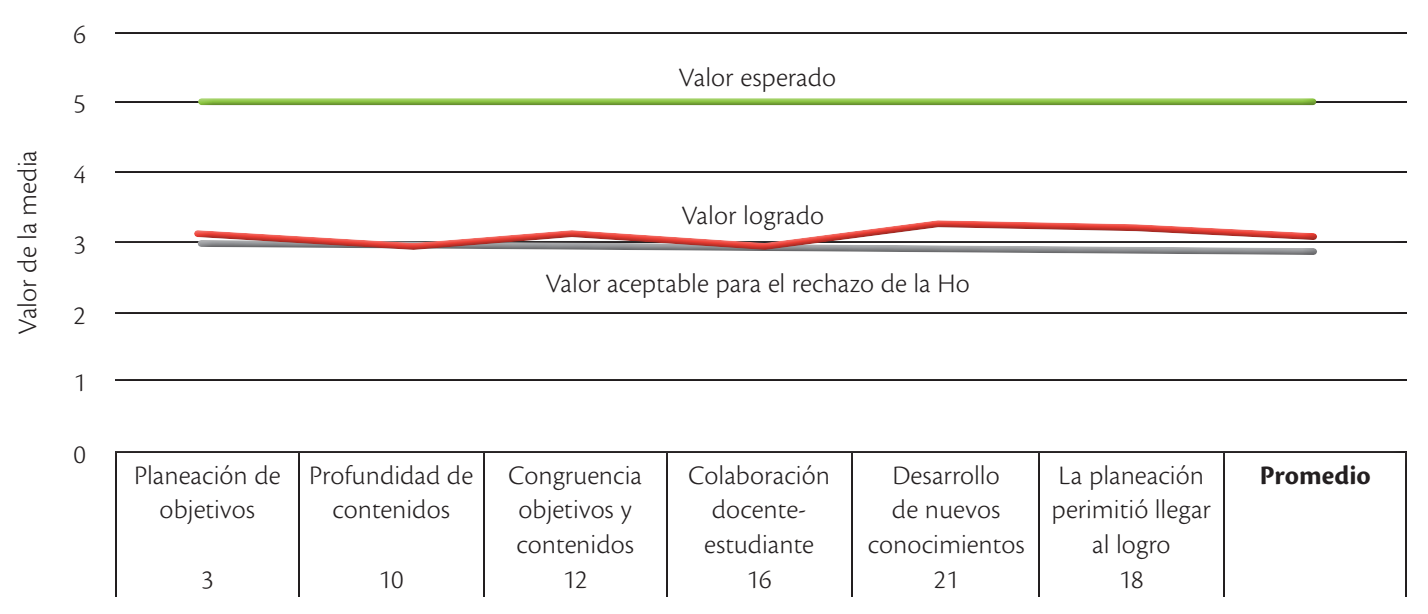

Fuente: propia de la investigación

Con el objeto de encontrar dificultades y facilidades otorgadas por los docentes en la integración de conocimientos entre las áreas y sub-áreas que componen la aplicación del Proyecto Integrador Político-Pedagógico de la Facultad de Enfermería de la Universidad del Estado de Rio de Janeiro, Brasil, comentan Ferreira et al. (2013) los factores en donde se presentan deficiencias contundentes en el desarrollo de la integración curricular, citan los siguientes aspectos: las características individuales y profesionales de los docentes y los comportamientos a veces inmaduros de los estudiantes en sus procesos de reflexión. Por lo que recomiendan a los alumnos - acerca de la deconstrucción de la visión fragmentada del currículo- la actualización continua en educación crítica y transformadora y reflexión, para que se sientan integrados a la colectividad operante de la enseñanza, y así poder contribuir eficazmente a la integración curricular; a los docentes recomiendan la constante aproximación a la propuesta pedagógica, para que la comprendan con claridad y con una visión crítica. Esta aproximación puede ocurrir a través de la participación activa de estos sujetos en las discusiones y planificaciones colaborativas de intercambio.

\section{II.2. Factores didácticos y pedagógicos en la gestión de la Al}

Las estrategias didácticas y pedagógicas utilizadas en el colegiado docente para llevar a cabo la actividad integradora son de notable importancia. La finalidad es que los docentes, en la aplicación de sus estrategias didácticas, logren que los estudiantes estén motivados y desarrollen las competencias genéricas, específicas y transversales de su profesión, en las actividades empleadas en el desarrollo del trabajo colaborativo. Los resultados obtenidos que determinan 
el comportamiento de las cinco variables que integran esta categoría de análisis involucran las estrategias didácticas y pedagógicas de integración, las de comunicación, las de motivación, las de trabajo colaborativo, las de participación y democracia en el trabajo en grupo y de interacción con los docentes; los resultados señalan, desde la perspectiva de los estudiantes, que por medio de la AI se alcanza un promedio de efectividad de 38\% para todas las variables de este grupo. Resultados que indican que el desarrollo de la actividad integradora está por debajo del $50 \%$ de efectividad, por lo que se tendrán que diseñar estrategias didácticas de mejora, ya que la AI tiene sólo seis años de implementación.

El valor más alto de la media de los promedios obtenidos corresponde a la variable motivación del estudiante para la realización de la AI, con un valor de 3.07, por arriba del promedio para el grupo de variables en esta dimensión, y en la prueba T Student, para una muestra se rechaza la hipótesis nula a un valor u de comparación de 2.5 , lo que indica el valor mínimo aceptable a una significancia de 0.000; el logro de las habilidades comunicativas obtuvo un valor promedio de 3.05 ; la participación y democracia lograda en el trabajo colaborativo y la estrategia didáctica y pedagógica utilizada con un valor promedio de 2.98, y por último el fortalecimiento del trabajo colaborativo, con un valor de 2.95; se determina a través del estadístico T Student, para una muestra fue mayor a 1.96 a un valor de u de comparación de 2.5 y una significancia de 0.000 , en la que se rechaza la Hipótesis nula (Ho), en su interpretación en la escala de significancia se encuentran en el nivel de mínimo aceptable (ver tabla 2 y gráfica 2).

En la opinión de Amadio et al. (2014) las expectativas acerca de la tarea de educar y el rol del docente han cambiado considerablemente en este nuevo siglo, ya que las políticas educativas favorecen principalmente el aprendizaje activo centrado en el estudiante, protagonista en el proceso de construcción y autorregulación de sus propios aprendizajes para satisfacer expectativas y necesidades. Las dimensiones cognitiva, ética y emocional están interrelacionadas, y de esta forma se favorece la comprensión y la aplicación de conocimientos, más que su propia acumulación, y se hace el mejor uso de las tecnologías de la información y la comunicación para lograr los objetivos diseñados.

Tabla 2. Factores didácticos y pedagógicos de influencia en la AI

\begin{tabular}{|c|c|c|c|c|c|c|}
\hline $\begin{array}{l}\text { Número } \\
\text { de Ítem }\end{array}$ & Variable & T Student & Significancia & Media & $\begin{array}{c}\text { Interpretación al } \\
\text { valor de comparación } \\
\text { rechaza la Ho }\end{array}$ & $\begin{array}{c}\text { Efectividad } \\
\text { Alcanzada en la } \\
\text { implementación }\end{array}$ \\
\hline 8 & Estrategia pedagógica integradora & $4.824^{*}$ & 0.000 & 2.98 & *Mínimo aceptable & $37 \%$ \\
\hline 16 & Se fortaleció el trabajo colaborativo (TC) & $4.355^{*}$ & 0.000 & 2.95 & *Mínimo aceptable & $37 \%$ \\
\hline 19 & Participación y democracia en el TC & $4.825^{*}$ & 0.000 & 2.98 & *Mínimo aceptable & $35 \%$ \\
\hline 20 & Habilidades comunicativas & $5.673^{*}$ & 0.000 & 3.05 & *Mínimo aceptable & $38 \%$ \\
\hline \multirow[t]{2}{*}{22} & Motivación & $5.069^{*}$ & 0.000 & 3.07 & *Mínimo aceptable & $40 \%$ \\
\hline & Promedio & & & 3.00 & & $38 \%$ \\
\hline
\end{tabular}

Fuente: propia de la investigación

Interpretación: Excelente ${ }^{* * * *} u=4.5$ a 5; Muy bueno ${ }^{* * * *} u=4-4.4 ;$ Bueno $^{* * *} u=3.5$ a 3.9; Regular $^{* *} u=3.0-3.4 ;$ Mínimo Aceptable $u=2.5-2.9$ 
Gráfica 2. Factores didácticos y pedagógicos de influencia en la AI

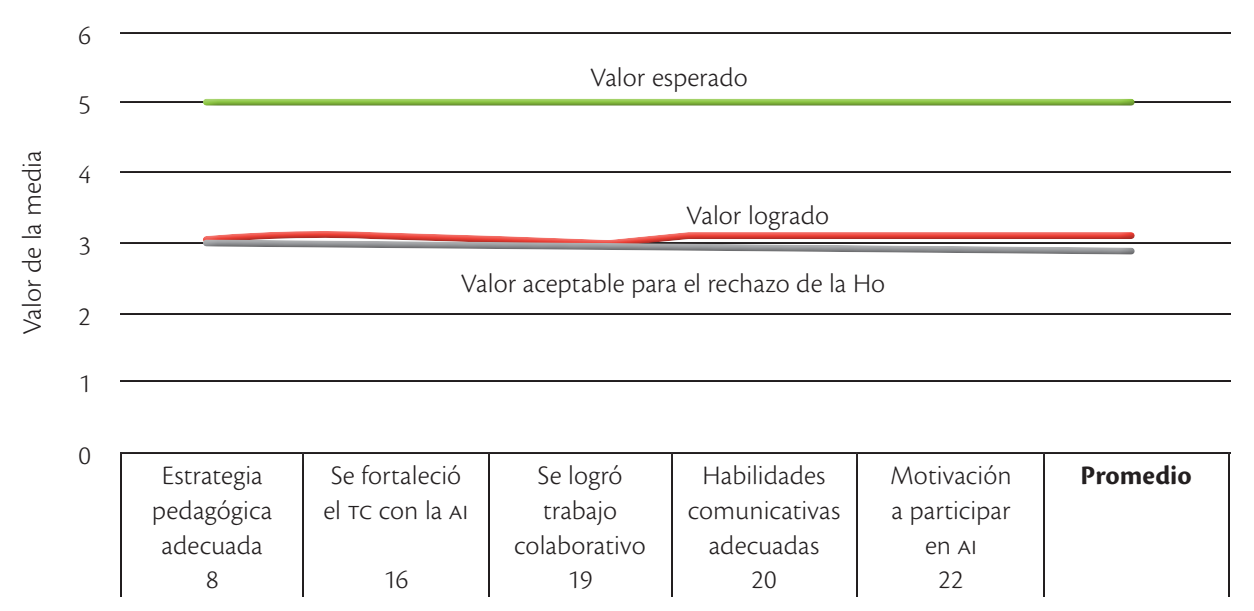

Fuente: propia de la investigación.

Los fundamentos ofrecidos por Amadio et al. (2014) refuerzan los resultados obtenidos en este rubro, ya que sostienen que el desarrollo de la AI y el logro de sus objetivos es de vital importancia, sin embargo, se pone de manifiesto la necesidad de fortalecer el trabajo del docente universitario en colegiado y el apoyo académico otorgado al estudiante en el desarrollo de esta actividad, para que los resultados sean un reflejo de que se cumple con los objetivos diseñados para la AI en el modelo Institucional MHIC, como un hecho consolidado y sobre todo para que el estudiante se sienta motivado y satisfecho en sus expectativas y necesidades de aprendizaje, en el desarrollo de sus competencias profesionales.

Los resultados en este apartado determinan una oportunidad de mejorar el desarrollo de la AI, se espera que el profesor refuerce en el estudiante la movilización de saberes, que esté ampliamente motivado y actúe con responsabilidad social para encontrar respuesta a los problemas que enfrenta en el ejercicio de su profesión de forma colaborativa. $\mathrm{Al}$ respecto destaca la opinión de Vélez (2013), quien dice que el estudiante, al irse percatando de la complejidad, diversidad y vinculación de saberes, estará consciente y capacitado para abordar las diferentes problemáticas por las que atraviesa en el campo de su especialidad, con una visión amplia que le otorga la AI, y un interés por colaborar con una disposición integradora, contextual y profunda en los problemas que enfrenta en el ejercicio de su profesión.

Por otro lado, atendiendo el rol del docente y el papel de la universidad como institución transformadora del contexto social, para Puñales y Fernández (2014), en la formación pedagógica universitaria se espera que el profesor comprenda el carácter social y multilateral de la enseñanza y con ello, su desempeño contribuya al mejoramiento de la educación, de los estudiantes y de la sociedad en general. El objetivo transformador de la personalidad del estudiante conlleva alcanzar niveles cualitativamente superiores en su desempeño profesional integral, ya que el estudiante que se especializa en estudios pedagógicos demanda una formación especial, por ser transmisor directo de la cultura y de los principios ideológicos propios de la profesión. 


\section{II.3. Las estrategias, recursos y mecanismos de evaluación en el desarrollo de la actividad integradora}

La actividad integradora como innovación educativa ante un nuevo modelo educativo curricular exige la implementación de mecanismos y herramientas innovadoras para la evaluación de las competencias adquiridas, la correcta implementación de una rúbrica puede ser la herramienta mediadora que cumpla esta función. La rúbrica es un instrumento idóneo para evaluar competencias, sobre lo desarrollado y aprendido, pues permite diseccionar las tareas complejas de manera graduada y operativa, lo que facilita que el estudiante sea consciente de hasta dónde llegan sus aprendizajes y cuál es el máximo nivel deseable en la entrega de sus tareas escolares, así como monitorear el desarrollo de la propia actividad autoevaluándose. Le facilita retroalimentación casi inmediata, ya que le permite acortar sustancialmente el tiempo de retorno, al ofrecer resultados cuantitativos y cualitativos basados en estándares conocidos previamente al desarrollo de la tarea.

Entre los resultados obtenidos a la valoración de esta dimensión de análisis se determina que las respuestas favorables a la utilización de las rúbricas para el desarrollo de la actividad integradora apenas alcanza un $40 \%$ de respuestas positivas en favor de su uso, ante el supuesto de tener como expectativa al menos el 50\% más uno de logrado, esto significa un reto para los docentes de mejorar en la parte operativa del diseño y aplicación de las rúbricas en la actividad integradora, hasta alcanzar una madurez tangible en el proceso de su aplicación.

Alcanza un $40 \%$ de efectividad el desarrollo de las estrategias, actividades y mecanismos de evaluación realizadas por los docentes y la propia autoevaluación que el estudiante realiza y le permite la retroalimentación en la AI. Un porcentaje de 42\%, 36\%, y $32 \%$ de efectividad alcanzada en favor de: lograr la autorregulación de sus aprendizajes, la metacognición, y la satisfacción en la calificación alcanzada respectivamente, valores por debajo del $50 \%$ de respuestas en favor de la implementación de los procesos de evaluación de la AI. De la misma forma, los resultados obtenidos a través de la prueba de Hipótesis por medio del estadístico T Student para una muestra, nos determina una valoración en el mínimo aceptable a un valor de comparación $\mathrm{u}=2.5$, resultados que coinciden con la valoración del algoritmo de frecuencia relativa de la respuesta positiva. Estos resultados, en la gestión del programa educativo, se presentan como factores críticos y de atención inmediata, y es necesario proponer estrategias de mejora para satisfacer estas necesidades y lograr que el alumnado sienta satisfacción en la parte operativa de los mecanismos de la evaluación de la AI en el colegiado de docentes, y en la utilidad de las rúbricas como mediadoras entre los procesos cognitivos que se llevan a cabo, asimismo, los mecanismos utilizados en estos procesos deben tener sobre todo una función retroalimentadora y formativa y no sólo determinan el esfuerzo realizado y el logro obtenido, plasmado en una cifra numérica (ver tabla 3 y gráfica 3 ).

En la opinión de Alsina (2013), la rúbrica es el instrumento cuya principal función es compartir criterios para la realización y guía de las tareas de aprendizaje y de evaluación que vinculan la interacción entre los estudiantes y el profesorado. En este sentido, la rúbrica señala las expectativas que los estudiantes y su profesorado esperan y comparten sobre una actividad o actividades, organizadas en diferentes niveles de cumplimiento: desde el menos aceptable hasta la resolución esperada, desde lo considerado como insuficiente hasta lo excelente y es así como debían de operar las estrategias de evaluación de la AI, de manera que se refleje el cambio educativo propuesto en el modelo curricular institucional

En la implementación de enfoques por competencias en la educación uno de los principales problemas, afirma Andrade (2008), está en la falta de vinculación de las competencias con las teorías pedagógicas en la formación de profesores, tanto en el aterrizaje de los modelos educativos como en el ejercicio de la 
evaluación en los estudiantes; al no comprender el cambio de enfoque del modelo educativo utilizado entre el diseño curricular y la práctica docente, se sigue haciendo lo mismo y se cometen los mismos errores, por lo que el desarrollo de innovaciones educativas en la práctica sólo cae en simulaciones y no se llega a los fines de lo diseñado, lo que exige que los mecanismos e instrumentos de evaluación sean prácticas de evaluación auténtica y representen una acertada retroalimentación de los saberes adquiridos en el estudiante.

Tabla 3. Factores de los procesos y herramientas de evaluación de impacto en la AI

\begin{tabular}{|c|l|c|c|c|c|c|}
\hline $\begin{array}{c}\text { Número } \\
\text { de İ́m }\end{array}$ & \multicolumn{1}{|c|}{ Variable } & T Student & Significancia & Media & $\begin{array}{c}\text { Interpretación al } \\
\text { valor de comparación } \\
\text { rechaza la Ho }\end{array}$ & $\begin{array}{c}\text { Efectividad } \\
\text { Alcanzada en la } \\
\text { implementación }\end{array}$ \\
\hline 1 & Utilidad rúbrica & $6.397^{*}$ & 0.000 & 3.12 & ${ }^{*}$ Mínimo aceptable & $42 \%$ \\
\hline 2 & Autoevaluar & $6.849^{*}$ & 0.000 & 3.10 & ${ }^{*}$ Mínimo aceptable & $40 \%$ \\
\hline 17 & Metacognición & $4.389^{*}$ & 0.000 & 2.93 & *Mínimo aceptable & $36 \%$ \\
\hline 11 & Autorregulación & $6.084^{*}$ & 0.000 & 3.08 & ${ }^{*}$ Mínimo aceptable & $42 \%$ \\
\hline 9 & Satisfacción & $3.806^{*}$ & 0.000 & 2.05 & ${ }^{*}$ Mínimo aceptable & $32 \%$ \\
\hline Promedio & & & $\mathbf{3 . 0 1 6}$ & & $\mathbf{3 8} \%$ \\
\hline
\end{tabular}

Fuente: propia de la investigación

Interpretación: Excelente ${ }^{* * * *} u=4.5$ a 5; Muy bueno ${ }^{* * * *} u=4-4.4 ;$ Bueno $^{* * *} u=3.5$ a 3.9; Regular** $u=3.0-3.4 ;$ Mínimo Aceptable $u=2.5-2.9$

\section{Gráfica 3. Factores de los procesos de evaluación de impacto en la AI}

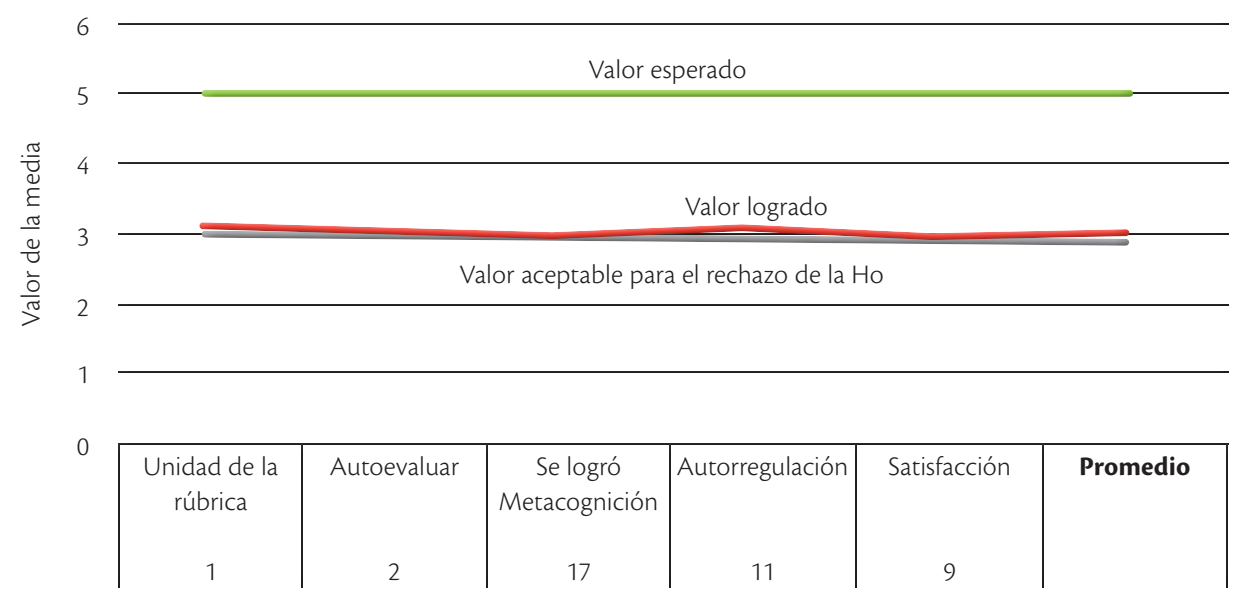




\section{Parte III. Sugerencias de los estudiantes para} mejorar las aportaciones de la actividad integradora en su formación profesional

Como respuesta a una de las preguntas abiertas se obtiene de manera cualitativa 300 respuestas que se clasificaron por su contenido y se interpretan desde su significado como áreas de oportunidad para la consolidación de la implementación de la AI y señalar acciones de mejora. A la pregunta ¿Qué sugieres para la mejora del desarrollo de la $\mathrm{AI}$ en beneficio del desarrollo de tus competencias profesionales y alcanzar el perfil de egreso?, se obtuvo, con el mayor porcentaje, el $40 \%$ en mejorar los procesos que se realizan en la evaluación de la AI; con 30\%, mejorar las estrategias de planeación de la AI entre docentes y estudiantes; con $20 \%$, sugieren que se mejoren las actividades colaborativas que realizan los docentes involucrados en el desarrollo de la AI, y con un $10 \%$, que la institución esté más comprometida en su desarrollo, ya que no cuentan con los espacios suficientes para su realización dentro del contexto de facultad y a algunos estudiantes les falta apoyo de equipo de cómputo y software especializado e internet; sus necesidades se ven reflejadas en el mismo orden de ideas a la valoración cuantitativa realizada.

Como se puede observar, estos resultados dejan ver una necesidad de los estudiantes; ellos esperan que sus profesores desarrollen de una mejor manera el trabajo colaborativo de gestión en la planeación y evaluación de los proyectos desarrollados en la AI (ver figura 1).

Figura 1. Áreas de oportunidad para el desarrollo de la Al

¿Qué sugieres para la mejora y aprovechamiento de la Al en beneficio del desarrollo de tus competencias profesionales y alcanzar el perfil de egreso?

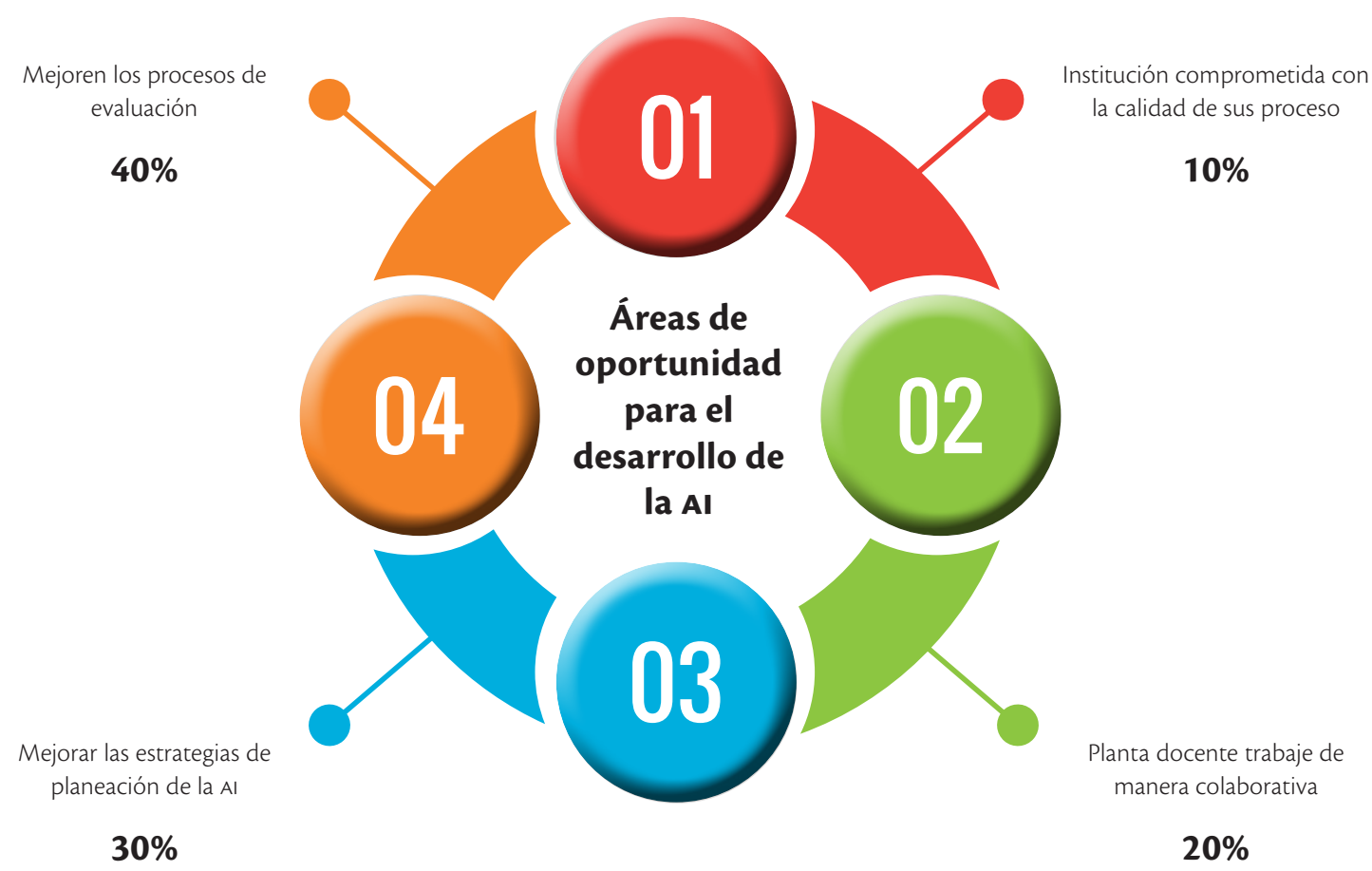

Fuente: propia de la investigación. 


\section{A manera de cierre}

Acerca de la evaluación realizada a la implementación y gestión de la AI en la formación del licenciado en Educación, como innovación curricular en la implementación del Modelo Educativo Humanístico Basado en Competencias, se puede concluir que: el porcentaje de estudiantes más desprotegido en apoyos de tecnología y de infraestructura para la realización de sus proyectos y tareas en la actividad integradora, aunque mínimo, está presente y son el grupo vulnerable en la realización de esta actividad, por lo que la universidad, como institución pública, tiene el compromiso de atender sus necesidades académicas y mejorar y optimizar los servicios académicos, escolares y de infraestructura, ya que influyen directamente en el desarrollo y formación de los estudiantes, con el fin de optimizar su efectividad como resultado final de la actividad sustantiva universitaria, ya que de alguna manera impactan en la formación profesional y finalmente en los indicadores de excelencia de los PE y de la institución.

El diseño y la planificación de la AI, para el logro de los objetivos de aprendizaje en los estudiantes y de su propia autorregulación en el ejercicio de la AI, tendrá que ser asertiva y congruente con la innovación curricular propuesta en el modelo educativo vigente. Así, se esperan acciones de mejora en el desempeño colaborativo de los docentes, en la asesoría, seguimiento, diseño de estrategias, instrumentos, procesos y mecanismos de evaluación; que éstos sean aplicadas de manera adecuada en la gestión de la AI, y se vean reflejados en los proyectos y en los informes finales propuestos por los estudiantes como resultado de la articulación entre la teoría, la práctica y la investigación.

Ante un nuevo modelo educativo como lo es el MHIC en la UATx, que exige innovaciones curriculares en el desarrollo de los PE y evaluaciones adecuadas a éstas, la rúbrica puede llegar a ser un potente motor de cambio metodológico como estrategia didáctica y pedagógica en la evaluación de los aprendizajes y en su vinculación a la práctica docente, para lograr en el estudiante la autonomía y autorregulación del aprendizaje. Lo que hace indispensable, en primer lugar, la formación de los docentes bajo este modelo y en trabajo colaborativo para el desarrollo de la AI; su participación, compromiso y reflexión sobre su desempeño en colegiado y de la interacción con los estudiantes, ante la gestión de estos procesos educativos. Que no sólo sirva para la medición de esfuerzos y logros alcanzados en la actividad desarrollada, expresados en un puntaje.

Los mecanismos e instrumentos de evaluación tienen que ser implementados de manera innovadora y asertiva, para satisfacer las necesidades de los estudiantes y su retroalimentación; que busquen impactar de manera positiva en el logro de saberes y su movilización en la realización de proyectos de investigación, en los que resuelvan problemáticas de su entorno con responsabilidad social universitaria y así desarrollar las competencias diseñadas en el perfil de egreso señaladas en los PE.

Lo observado y evaluado en esta investigación muestra la efectividad alcanzada en las estrategias implementadas, refleja aciertos, logros y necesidades por satisfacer en los estudiantes; si bien se observan algunas incongruencias y contradicciones de lo requerido en la gestión de la AI, se detectan áreas de oportunidad para fundamentar y enriquecer las estrategias pedagógicas empleadas como innovación educativa en las revisiones curriculares de los PE, como lo es el caso de la AI.

Es importante concluir que, aproximadamente siete años después de la implementación del nuevo modelo universitario (MHIC) y sus innovaciones curriculares, como el desarrollo de la AI, apenas se ha alcanzado el 50\% de efectividad. La revisión curricular sugerida por los organismos acreditadores y certificadores para los programas educativos de licenciatura es, en el corto plazo, cinco años después de su implementación.

Solange (2005) y Sanders (2012) señalan que, a pesar de las dificultades en la implementación de 
una innovación curricular desde la pedagogía de la integración, y a través de la investigación educativa, los docentes coinciden en que los fines que persigue la integración escolar de vincular la parte cognitiva con otras áreas de desarrollo aunadas a la tecnología son loables y válidas de poner en práctica, y que la experiencia en el desarrollo de la actividad ha permitido perfeccionar esta práctica, dando mayor seguridad a los estudiantes para adquirir las competencias en este proceso formativo.

Desde otra perspectiva, la visión en que se destaca la integración como positiva y en la cual se han construido las condiciones para su implementación exitosa, destaca una cultura escolar con capacidad de innovación y disposición al cambio educativo. Por lo que se concluye que en las estrategias de implementación para la gestión de una innovación curricular, se debe prever la formación del cuerpo docente en la apropiación del nuevo modelo educativo; la fundamentación de las mismas innovaciones que éste conlleva, y la adecuación de la tecnología de la información y conocimiento y de la infraestructura, que sean propias en su conjunto a las necesidades de los estudiantes y académicos en el contexto institucional, y congruentes con el desarrollo de las innovaciones. Los cambios educativos requieren de fundamentos teóricos y acciones pedagógicas y didácticas vinculadas de manera práctica, y alineadas a las políticas institucionales y nacionales de manera congruente, para dar respuesta a las necesidades sociales emergentes en nuestro contexto de desarrollo y de nuestro país, en una interacción global, para responder con ética y responsabilidad social universitaria. 


\section{Referencias}

Alsina, Josep (2013), “Qué es una rúbrica? ¿Por qué y cómo se utiliza?", en J. Alsina (coord.), Rúbricas para evaluación de competencias, Barcelona, Universidad de Barcelona/OCTAEDRO, ICE (Guadernos de Docencia Universitaria 26, http://hdl.handle.net/2445/145046 [Consulta octubre 2017].

Andrade, Rocío (2008), "El enfoque por competencias en educación”,Ide@sCONCRTEG, año3,núm.39,<http:// www.researchgate.net/publication/267553115_ El_enfoque_por_competencias_en_educacion $>$ [Consulta: noviembre de 2017].

Amadio, Massimo, Renato Opertti y Juan Tedesco (2014), "Un currículo para el siglo XXI: Desafios, tensiones y cuestiones abiertas", en Investigación y Prospectiva en Educación UNESCO [Documentos de Trabajo ERF, No. 9], París, disponible en <http://www.ibe.unesco.org/ sites/default/files/resources/229458s.pdf> [Consulta: octubre de 2017].

Cevallos, Gabriel, Evelyn Alcívar, Cristobal Rey y Manuel Roa (2016), "Proyectos integradores de saberes como estrategia didáctica de aprendizaje en los estudiantes del Instituto Tecnológico Superior Julio Moreno Espinosa", Revista Atlante: Cuadernos de Educación y Desarrollo, disponible en: <http://www.eumed.net/ rev/atlante/2016/03/conocimiento.htm> [Consulta: septiembre de 2017].

Coll, César (2007), "Las competencias en la educación escolar: algo más que una moda y mucho menos que un remedio", Aula de Innovación Educativa, núm. 161, 34-39, <https://pasionytinta.files.wordpress.com/2013/04/ coll-competencias-en-educacic3b3n-escolar.pdf $>$ [Consulta: enero de 2017].

Cruz, Rodolfo y Gabriela Croda (20017), "Concepciones sobre innovación educativa: elementos para su teorización [C-0580]", memoria electrónica del COMIE 2017, <http://www.comie.org.mx/congreso/ memoriaelectronica/v14/doc/0580.pdf> [Consulta: septiembre de 2017].

Ferreira, Iraneide, Norma MafraI, Valéria Dantas de
Oliveira, Marcela Fernandes Costa y Lucia Garcia (2013), "Projeto político-pedagógico: fragilidades e potencialidades vividas por docentes universitários de enfermagem", Rev. enferm. UERf, Rio de Janeiro, 2013 jul./ set.; vol. 21, núm. 3, pp. 361-365, disponible en: <https:// www.e- publicacoes.uerj.br/index.php/enfermagemuerj/ article/view/7524/5444> [Consulta: enero de 2018].

Gaeta, Martha (2006), "Estrategias de autorregulación del aprendizaje: contribución de la orientación de meta y la estructura de metas del aula", Revista Electrónica Interuniversitaria de Formación del Profesorado, vol. 9, núm. 1, pp. 1-8, disponible en: <https://www.redalyc.org/comocitar. oa?id=217017165001 > [Consulta: enero de 2018].

Hewitt, Nohelia (2007), "El proyecto integrador: una estrategia pedagógica para lograr la integración y la socialización del conocimiento", Psicología. Avances de la disciplina, vol. 1, núm. 1, enero-junio, 2007, pp. 235240, disponible en: <http://www.redalyc.org/articulo. oa? id=297224869006> [Consulta: febrero de 2018].

Kalimullin Aydar, Olga Korshunova y Julia KoinovaZoellner(2016), "Balance pedagogy as a methodological base for integrative-differentiated teaching of rural students", International Fournal of Environmental E Science Education, vol. 11, núm. 7, pp. 1539-1550 (Originally published by Look Academic Publishers in IJESE, ISSN: 1306-3065), <https://files.eric.ed.gov/fulltext/ EJ1 114432.pdf> [Consulta: diciembre de 2017].

Núñez, José, Julio González-Pineda, Pedro Rosario y Paula Solano (2006), "Autorregulación del aprendizaje: un nuevo desafío del estudiante de enseñanza superior", Infocop, 21/09, disponible en <http://www.infocop.es/view_ article.asp?id=1039> [Consulta: diciembre de 2017].

Ortiz, Serafín (2015), La formación humanista en la educación superior: Modelo humanista integrador basado en competencias, México, Editorial GEDISA.

Pareja, Giraldo Alexander (s/f), "Proyecto integrador", disponible en: <https://www.scribd.com/document/ 139173033 / PROYEGTO-INTEGRADOR > [Consulta: enero de 2017]. 
Parra, Blanca y Jaime Pinzón (2013), "Proyecto integrador como estrategia formativa para el fortalecimiento de competencias específicas y transversales en la facultad de ingeniería", Ponencia presentada en el Foro Mundial de educación en Ingeniería, disponible en: <https://www. acofipapers.org/index.php/acofipapers/2013/paper/ viewFile/105/32> [Consulta: febrero de 2018].

Pérez, Ma. Victoria, Marco Valenzuela, Alejandro Díaz, Julio González-PiendayJoséNúñez(2013), "Dificultades de aprendizaje en estudiantes universitarios de primer año", Atenea, núm. 508, 2013, pp. 135-150, ISSN 0716-1840, disponible en: <http://www.redalyc.org/ articulo.oa?id=32829669010> [Consulta: octubre de 2017].

Puñales, Lucía y Omara Fernández (2014), "El proceso de formación del estudiante de la carrera licenciatura en educación primaria", Atenas, vol. 3, núm. 27, pp. 85-99, disponible en: <https://www.redalyc.org/ comocitar.oa? id $=478047203007>$ [Consulta: enero de 2018].

Roegiers, Xavier (2010), Una pedagogía de la integración. Competencias e integración de los conocimientos en la enseñanza (Tr. Juan José Utrilla), México, Fondo de Cultura Económica.
Sanders, Mark (2012), "Integrative stem education as best practice", en H. Middleton (ed.), Explorations of best practice in technology, design $\mathcal{E}$ engineering education, Australia, Griffith Institute for Educational Research, <https://vtechworks.lib.vt.edu/bitstream / handle/10919/51563/SandersiSTEMEdBestPractice. pdf ? sequence $=1$ \&isAllowed $=\mathrm{y}>$ [Consulta: enero de 2018].

Solange, Tenorio (2005), "La integración escolar en Chile: perspectiva de los docentes sobre su implementación", Revista Electrónica Iberoamericana sobre Calidad, Eficacia y Cambio en Educación, vol. 3, núm. 1, disponible en <http://www.ice.deusto.es/rinace/reice/vol3nl_e/ Tenorio.pdf $>$ [Consulta: enero de 2018].

Vélez, Waldemiro (2013), "Integración de saberes y formación integral en los estudios generales del siglo XXI", en Simposio Internacional de Estudios Generales, 6, 7 y 8 de noviembre, Puerto Rico, Universidad de Puerto Rico, Recinto de Ríos Piedras/Red Internacional de Estudios Generales (RIDEG), disponible en <http:// www.rideg.org/wp-content/uploads / $2014 / 04$ / Integraci $\% \mathrm{C} 3 \% \mathrm{~B} 3 n$-de-saberes-y-formaci $\% \mathrm{C} 3 \% \mathrm{~B} 3 n$ integral-en-los-estudios-generales-del-siglo-XXI.pdf> [Consulta: enero de 2018]. 